\title{
Evaluation des risques hydro-climatiques et environnementaux liés à l'occurrence de l'ulcère de Buruli dans la région de la Marahoué (Côte d'Ivoire)
}

\author{
Assikohon Pulchérie GOUZILE ${ }^{1 *}$, Gneneyougo Emile SORO ${ }^{2}$, \\ Zilé Alex KOUADIO ${ }^{3}$ et Tié Albert GOULA BI ${ }^{4}$ \\ ${ }^{I}$ Unité de Formation et de Recherche en Science et Gestion de l'Environnement, Université Nangui Abrogoua, \\ 02 Bp 801 Abidjan, Côte d'Ivoire. \\ ${ }^{2}$ Unité de Formation et de Recherche en Science et Gestion de l'Environnement, Université Nangui Abrogoua, \\ 02 Bp 801 Abidjan, Côte d'Ivoire. \\ ${ }^{3}$ Unité de Formation et de Recherche Environnement, Université Lorougnon Guédé, BP150 Daloa, \\ Côte d'Ivoire. \\ ${ }^{4}$ Unité de Formation et de Recherche en Science et Gestion de l'Environnement, Université Nangui Abrogoua, \\ 15 Bp 950, Abidjan, Côte d'Ivoire. \\ *Auteur correspondant ; E-mail : gouzilepul@yahoo.fr, Tel : 0022557062090
}

\section{RESUME}

Ce travail aborde un problème de santé dû à l'ulcère de Buruli en zone pré-forestière (zone de contact forêt- savane) dans la région de la Marahoué en Côte d'Ivoire. Cette maladie est due à une mycobactérie : la Mycobacterium ulcerans. L'objectif de cette étude est de déterminer des zones à risque à la Mycobacterium ulcerans afin de pouvoir protéger les populations vulnérables. Pour y parvenir, une approche combinée de systèmes d'information géographique et l'analyse multicritère a été adoptée. La cartographie des zones potentielles à risque à l'ulcère de Buruli révèle que le sud de la région présente un risque élevé de la transmission l'ulcère de Buruli. Ce risque élevé est lié à trois types d'indicateurs regroupant les facteurs. L'indicateur lié aux facteurs climatiques (précipitation, température et humidité relative) couvre 41,54\% de superficie. L'indicateur lié aux facteurs environnementaux tels que l'indice de végétation (NDVI), l'indice d'humidité du sol (NDWI), l'altitude, l'hydrographie, et à la population, couvre 55,24\% et l'indicateur lié à l'occupation du sol couvre $3,22 \%$ de la superficie. Par ailleurs, les résultats montrent que $57,17 \%$ de la superficie totale de la région est favorable à la transmission de l'ulcère de Buruli. Dans l'ensemble, les indicateurs climatiques, environnementaux, la population et l'occupation du sol constituent des facteurs de risques associés à l'émergence de l'ulcère de Buruli.

(C) 2016 International Formulae Group. All rights reserved.

Mots clés : Cartographie, risque, ulcère de Buruli, analyse multicritère, Côte d'Ivoire,

\section{Assessment of hydro-climate and environmental risks associated with the Buruli ulcer occurrence in Marahoué region (Côte d'Ivoire)}




\begin{abstract}
This study discusses a health care issue caused by Burili ulcers in pre-forested areas (contact area, forest-savanna) in Marahoué region of Côte d'Ivoire. That disease is caused by a mycobacterium: the Mycobacterium ulcerans. The aim of this work was to determine risk areas to Mycobacterium ulcerans in order to protect vulnerable populations. To achieve this objective, a combined approach of GIS and multi-criteria analysis has been proposed. The mapping of potential risk areas shows that the southern region has a high risk of transmission of Buruli ulcer. This risk is linked to three types of indicators grouping the factors. The indicator related to climatic factors (precipitation, temperature and relative humidity) covers $41.54 \%$ of the area. The indicator linked to environmental factors (vegetation index, humidity index of soil, altitude and hydrography) and population covers $55.24 \%$ and the indicator for the land use covers $3.22 \%$ of the area. Furthermore, the results show that $57.17 \%$ of the total area of the region are favorable for the transmission of Buruli ulcer. Overall, climate and environmental indicators, population and land use are risk factors for the emergence of Buruli ulcer.

(C) 2016 International Formulae Group. All rights reserved.
\end{abstract}

Keywords: Mapping, risk, Buruli ulcer, multi-criteria analysis, Côte d'Ivoire

\section{INTRODUCTION}

L'ulcère de Buruli est une infection due à une mycobactérie environnementale appelée Mycobacterium ulcerans qui se développe dans les systèmes aquatiques (Darie, 2003). Elle est classée troisième après la lèpre et la tuberculose et constitue ainsi un problème majeur de santé publique (Kibadi, 2005). Depuis 1980, elle est devenue une cause de souffrance humaine (OMS, 2004). Elle est signalée dans près de 33 pays situés principalement en zones tropicales ou subtropicales avec 2200 nouveaux cas chaque année (OMS, 2015). L'Afrique occidentale semble véritablement touchée par cette maladie depuis deux décennies, avec une recrudescence inquiétante au cours de la présente décennie, malgré sa très faible contagiosité (Kanga et Kacou, 2000). La Côte d'Ivoire, est un pays endémique à cette pathologie (Brou et al., 2008 ; Fener, 2015). Cette infection occupe le deuxième rang des mycobactériose en Côte d'ivoire après la tuberculose et avant la lèpre (Kanga et Kacou, 2000). La première observation a été faite en 1978 chez un jeune Français ayant fréquenté les abords du lac Kossou, situé dans la région de la marahoué qui est la zone d'étude. Depuis lors, l'incidence de la maladie a augmenté dans ce pays avec 2000 nouveau cas chaque année (Fener, 2009). Bien que la localisation dans les écosystèmes et le mode de transmission ne soient pas encore bien caractérisée, l'épidémiologie de cette infection semble liée aux conditions environnementales et ses modifications (Marsollier et al., 2003). En dépit de ces incertitudes sur le lien environnement-santé, plusieurs études ont souligné l'existence d'un lien environnementale caractérisé par la présence des fleuves, lacs artificiels ou naturels, rivières à débit lent, mares et zones marécageuses, systèmes d'irrigation, zones agricole à proximité de l'eau (Kanga et Kacou, 2000 ; Brou et al., 2006). Certains auteurs insistent même sur la variation climatique et les modifications environnementales (inondation etc...) comme facteurs favorisant le développement de $M$. ulcerans et à sa transmission à l'homme (Mouchou et Mougin, 2009 ; Baquey, 2014). L'objectif de cette étude est decartographier les zones de potentiel risque de l'ulcère de Buruli dans la région de la Marahoué en Côte d'Ivoire en vue d'une lutte plus efficace contre cette pathologie. Cette approche utilise certains outils comme la cartographie, la télédétection et les SIG et permet de mieux 
cibler les populations et les espaces à risque (Somé, 2010). Elle est la plus appropriée pour évaluer les risques sanitaires en association avec l'environnement en ce sens qu'elle combine des méthodes épidémiologiques, les statistiques et les techniques des systèmes d'information géographique (SIG) (Beale et al., 2008). Ainsi, les SIG sont les mieux indiqués pour faire ressortir les tendances, les corrélations et les interactions entre la maladie et les facteurs favorisant la distribution de la maladie (Kouamé et al., 2011). Ils permettent d'identifier les zones potentiellement à risque d'une maladie. En Côte d'Ivoire dans la région de l'Agnéby, les techniques des SIG ont été utilisées pour déterminer les facteurs de répartition de la Bilharziose et des Géohelminthes (Kouamé, 2010).

\section{MATERIEL ET METHODES \\ Matériel \\ Données climatiques}

Elles concernent les précipitations, la température maximum (max), la température minimum (min) et l'humidité relative sur la période de 1981 à 2013. Elles ont été à notre disposition par la Direction de la Météorologie Nationale (SODEXAM). Les données de précipitation, de température max, de température min et d'humidité relative proviennent des stations synoptiques de Daloa, Yamoussoukro, la station pluviométrique de Bouaflé et le complexe sucrier de la SUCAF de Zuénoula. Ces données sont utilisées pour estimer le risque lié aux facteurs climatiques.

\section{Données environnementales}

Elles sont composées d'une image satellitaire ETM+ (Enhancement Thematic Mapper plus) de Landsat 7 à résolution spatiale de 30 m, Scène 197/055 zone 29 étendu à la zone 30 acquise le 12 décembre 2003. Elle a servi à la réalisation des cartes d'indice de végétation (NDVI) et d'humidité $\mathrm{du}$ sol (NDWI). Le modèle numérique d'altitude (MNT clip) MNT-SRTM3 (Schuttle Radar Topography Mission) d'une résolution de 30 ma été utilisé pour établir la carte du modèle numérique d'altitude (MNA) et la carte du réseau hydrographique

\section{Outils}

Le traitement de ces données a nécessité l'utilisation des logiciels suivent:

- ArcGis 10. Pour la cartographie des zones de risque.

- Envi 4.7 de RSI (Research Système Incorporation) a été utilisé pour le traitement de l'image acquise et l'occupation du sol.

\section{Méthodes}

La cartographie des zones de risque potentiel à l'ulcère de Buruli nécessite des données d'ordre climatiques, environnemental et le type d'occupation de sol. Ces données sont les critères qui conditionnent l'émergence et l'existence des foyers endémiques à l'ulcère de Buruli (Darie, 2003 ; Kanga et al., 2004 ; Brou et al., 2006 ; Christinet et al., 2012 ; Baquev, 2014). La démarche consiste en la classification et la standardisation des critères, en la pondération des critères et en une agrégation suivant la démarche multicritère (Youan Ta et al., 2011; Mangoua et al., 2014).

\section{Choix des critères de décision}

Les facteurs ou critères utilisés pour identifier les zones potentielle au risque à l'ulcère de Buruli, sont composé du couvert végétal, de l'humidité du sol, l'altitude, les points d'eau, des zones irriguées et drainées ; des précipitations et la température. La Mycobacterium ulcérans bactérie responsable de l'ulcère de Buruli, prolifère dans les zones agricoles à proximité des points d'eau, les zones marécageuses, les cours d'eau à écoulement lent, les lacs naturels ou artificiel etc. Ainsi, les paramètres favorisant l'existence des foyers de l'ulcère de Buruli comprennent trois indicateurs de vulnérabilité que sont: la vulnérabilité climatiques, environnementale et l'occupation du sol.

\section{Vulnérabilité climatique}

L'indicateur vulnérabilité climatique traduit l'ensemble des facteurs climatiques qui favorisent la présence de l'ulcère de Buruli. Il 
résulte de la combinaison linéaire des cartes de précipitation, de température max et min et de l'humidité relative obtenues par la technique d'interpolation Inverse Distance Weigthing (IDW), de l'extension géostatitica analyst d'ArcGIS.10.

\section{Vulnérabilité environnementale} L'indicateur vulnérabilité environnementale conditionne les facteurs environnementaux qui favorisent le développement du germe. Il résulte de la combinaison linéaire des cartes d'indice de végétation (NDVI), d'indice d'humidité du sol (NDWI), de l'altitude et de la distance en tout point d'eau (hydrographie). Les cartes d'indice de végétation et d'humidité du sol sont obtenues à partir des traitements de l'image ETM+ de Landsat par composition $\begin{array}{llll}\text { coloré sur } & \text { ENVI }\end{array}$ L'extractiondelazoned'études'esteffectuée par masque binaire sur une scène de Landsat7. L'indice de végétation a été déterminé par la formule de «différence pondérée » plus la valeur tend vers 1 plus le couvert végétal est dense et chlorophyllement actif, plus l'indice est faible moins il y a de végétation. La formule de calcul de cet indice est :

$N D V I=\frac{P I R-R}{P I R+R}$

$=\frac{P I R-M I R}{P I R+M I R} N D W I=\frac{T M 4-T M 5}{T M 4+T M 5}$ Avec PIR le proche infrarouge et le $\mathrm{R}$ le Rouge. Dans le cas des images ETM+ de LANDSAT que nous avons utilisé, le PIR correspond à la bande $\mathrm{ETM}+3$ et $\mathrm{R}$ à la bande ETM+4. Par ailleurs, l'indice d'humidité du sol est obtenu par la formule suivante.

L'hydrographie et l'altitude sont obtenues à parti du modèle numérique d'altitude (MNT clip) MNT-SRTM3 (Schuttle Radar Topography Mission). La carte de densité de population est obtenue par interpolation Inverse des Distance ou Inverse Distance Weigthing (IDW), de l'extension géostatitica analyst d'ArcGIS.10. Des valeurs de la densité de population résultant du rapport de la population sur la superficie de chaque localité de la région.

\section{Occupation du sol}

Tous comme la végétation et l'humidité du sol, la carte d'occupation du sol est déterminée à partir des traitements d'image ETM+ de Landsat par composition coloré sur ENVI 4.7. L'extraction de la zone d'études est effectuée par masque binaire sur une scène de Landsat 7. La composition colorée consiste à afficher simultanément à l'écran trois bandes d'images dans les canaux de base (Rouge/Vert/Bleu) et faciliter l'extraction des informations. Dans le cadre de cette étude, la composition colorée des bandes 4-5-3 de Landsat TM et ETM+ a été utilisée parce qu'elle présente la meilleure discrimination des types d'occupation du sol.

\section{Classifications et Standardisation des critères de décision}

Les critères de décision identifiés ont été subdivisés en différentes classes représentant soit un milieu particulier, soit un intervalle de confiance. Deux classes qualifiées de risque faible et risque élevé ont été retenues pour chaque critère dans l'optique d'une meilleure interprétation (Kouamé et al., 2011). Par la suite, les différentes classes de chaque critère ont été standardisées en fonction de leur influence particulière sur la pathologie. Ces classes ont été codifiées (codification numérique). La combinaison des indicateurs a donné les cartes du risque global de l'ulcère de Buruli. La classification et la standardisation des critères de décision sont consignées dans le Tableau 1.

\section{Pondération des critères}

Les critères de décision sont pondérés suivant la méthode de combinaison linéaire basée sur la technique de comparaison par paire selon le processus d'analyse hiérarchique (Analytic Hierarchy Process, AHP) de Saaty (1977). Elle est développée par Meyer-Waarden et al. (2005) et utilisé par Youan Ta et al. (2011) et Yao et al. (2014). Cette méthode génère des coefficients de pondération standardisée dont la somme est 
égale à 1. Elle consiste à tout d'abord à une comparaison binaire de l'importance relative de tous les éléments appartenant à un même niveau hiérarchique par rapport à l'élément du niveau immédiatement supérieur selon l'échelle numérique proposé par Saaty (Tableau 2). Ensuite, configurer une matrice carrée réciproque formée par les évaluations des rapports des poids ( $\mathrm{K} \times \mathrm{K})$, $\mathrm{K}$ étant le nombre d'éléments comparé. On obtient de cette façon :

$$
a_{j i}=\frac{1}{a_{i j}}
$$

Avec a=aij et aij=1 (valeur réciproque) où a est la valeur de chaque facteur et $\mathrm{i}$ et $\mathrm{j}$ constituent respectivement les lignes et les colonnes (Saaty, 1977).En fin elle permet de déterminer les poids de chaque critère par la méthode approchée de calcul des vecteurs propres (Vpi). Les vecteurs propres sont obtenus en calculant leur moyenne géométrique par ligne. Le Vpi est déterminé par la formule suivante :

$$
V_{\rho i}=\sqrt[n]{\prod_{i=1}^{n} N_{i}}
$$

Avec: Vpi vecteurs propre de chaque critère, $\mathbf{N} i$ Valeur de chaque critère. Le coefficient de pondération Wi est déterminé comme suite

$$
W_{i}=\frac{v_{\rho i}}{\sum_{i=1}^{n} v_{\rho i}}
$$

L'échelle numérique proposée par Saaty est représenté dans le Tableau 2.

La matrice de comparaison binaire et les coefficients de pondération des critères pour chaque critère et pour les trois indicateurs sont représentés par le Tableau 3.

\section{Evaluation des critères de décision}

Diverses méthodes ont été appliquées pour l'évaluation des critères de décision dans l'analyse multicritères. Dans cette étude, nous avons retenu, la méthode d'agrégation complète par pondération utilisée par Martin et al. (2004). Elle consiste en une multiplication de chaque critère ou indicateur par son coefficient de pondération et ensuite en additionner les résultats pour produire un indice d'aptitude selon l'équation suivante :

$\mathrm{S}=\sum_{i=1}^{n}$ wixi
Avec: $\mathrm{S}$ : le résultat final; $\mathrm{Wi}$ : le poids des critères $\mathrm{i} ; \mathrm{Xi}$; valeur standardisé du critère $\mathrm{i}$

Cette méthode a été utilisée pour l'indicateur vulnérabilité climatique et environnemental. La réalisation de la carte de risque de l'ulcère de Buruli consiste à reporter dans l'espace, les différentes valeurs issues de l'addition des valeurs standardisées et pondérées de chaque indicateur intervenant ajouté à l'occupation du sol. Une reclassification des critères a permis d'obtenir des cartes thématiques à deux classes que sont le risque faible et le risque élevé. Le nombre de classe est fixé à deux pour une meilleure lisibilité et une bonne interprétation de la carte.

\section{Analyse de l'incertitude et l'erreur de carte}

L'analyse consiste à calculer les incertitudes de la moyenne des différents paramètres des principaux indicateurs de puissance et de la vulnérabilité. L'incertitude est calculée par l'équation suivante :

$$
\Delta \bar{x}=\frac{\sigma}{\sqrt{m}}
$$

Avec : $\Delta x:$ l'incertitude de la moyenne de la série de données; $\Sigma$ : l'écart-type de la série de données $\mathrm{m}$ : le nombre de données.

Pour déterminer l'intervalle de confiance, un facteur d'expansion (K) est ensuite calculé. La détermination de ce facteur est basée sur le principe de calcul statistique de l'incertitude étendue. Le facteur K permet la définition d'un intervalle de portée suffisante pour avoir une grande confiance dans les résultats. L'expression de ce facteur est la suivante :

$K=\frac{|E-\bar{x}|}{\sigma}$

Avec: $\mathrm{K}$ : facteur d'expansion; $\mathrm{E}$ : valeur extrême de la série statistique. Elle peut être le maximum ou le minimum de la série; $\sigma$ : écart-type de la série.

Le niveau de confiance des différents paramètres a été déduit à partir des différentes valeurs de $\mathrm{K}$. ainsi, $\mathrm{K}=1$ pour le niveau de confiance de $68 \%, \mathrm{~K}=2$ pour le niveau de confiance de $95 \%$ et $\mathrm{K}=3$ pour le 
niveau de confiance de $99 \%$ (Yao et al., 2014).

\section{RESULTATS}

\section{Occupation du sol}

La cartographie de l'occupation du sol de la zone d'étude a permis de déterminer cinq classes (eau, forêt dégradée, culture/jachère, Forêt dense et sol nu et habita) en fonction de leur degré de vulnérabilité. La forêt dense est observée pratiquement sur toute la zone d'étude en diverse points. Cependant, elle est plus présente à l'ouest de la zone d'étude précisément au niveau du parc de la Marahoué. Elle occupe $44,8 \%$ de la superficie totale. La forêt dégradée, les cultures et jachères, le sol nu et l'eau occupent respectivement $22,7 \% ; 22,90 \% ; 9,1 \%$ et $0,5 \%$ de la région (Figure 1).

\section{Risque de l'ulcère en fonction des facteurs climatiques}

La carte de risque de l'ulcère de Buruli en fonction des facteurs climatiques dans la région de la Marahoué est représentée par la Figure 2. L'analyse de cette carte montre deux classes.

-la classe de degré de risque faible couvre $58,46 \%$ soit plus de la moitié de la zone d'étude. Cette classe est présente à l'extrême nord de Zuénoula, à l'est de la zone d'étude à proximité du barrage de Kossou et certaine sous-préfecture de Bouaflé. Elle est caractérisée par des faibles précipitations qui varient entre 1096 et $1152 \mathrm{~mm}$, des températures max très élevées $\left(35^{\circ} \mathrm{C}\right)$ et une humidité relative inférieur à $80 \%$. Cette zone ne favorise par la prolifération de la maladie. Toutefois quelques zones endémiques ont pu être détectées.

-la classe de risque élevé couvre $41,54 \%$ de la zone d'étude. Cette zone s'étend de l'extrême nord-ouest de la région et arrive jusqu'aux sud en passant par le centre ouest. Elle touche aussi le parc de la Marahoué et est caractérisée par des précipitations élevé et températures maximale $\left(30{ }^{\circ} \mathrm{C}\right)$ avec une humidité relative élevée
(>75\%). Ces facteurs favorisent la présence de forêt, des points d'eau (cours d'eau, lac, basfond rizicole....) qui sont des critères favorisant le risque de l'ulcère Buruli. Cette zone est plus à risque car les facteurs climatiques présentent des conditions favorables à la présence de zone humide pouvant ainsi entraîner la distribution de la pathologie.

\section{Risque de l'ulcère de Buruli en fonction des facteurs environnementaux}

Le risque en fonction des facteurs environnementaux traduit la vulnérabilité à l'ulcère de Buruli due aux variables écologiques telles que: le couvert végétal, l'humidité du sol, la distance aux points d'eau (hydrologie), l'altitude et la densité de population. Le vecteur et le parasite (Mycobacterium ulcerans) de la maladie se développent dans ces facteurs.

La carte de risque en fonction des facteurs environnementaux ou écologiques présente deux classes (Figure 3).

- la classe de risque faible couvre $44,76 \%$, soit moins de la moitié de la zone d'étude. Elle se situe dans la partie nord de la région, et dans certaines localités du département de Bouaflé (Tibiéta, Bouafla...). Elle est caractérisée par une dégradation du couvert végétal avec présence de culture vivrière et une diminution du taux d'humidité du sol, en plus, les localités sont éloignées des points d'eau et les altitudes sont élevées. Cette zone ne favorise par la prolifération de la maladie par contre quelques zones endémiques peuvent être détectées.

- la classe de degré de risque élevé couvre $55,24 \%$ de la région et se situe dans le sud de la région; dans les départements de Sinfra et de Bouaflé, dans la zone du parc nationale de la Marahoué et à proximité du lac de Kossou. Elle est caractérisée par la présence d'un couvert végétal plus intense caractérisé par la forêt et de cultures industrielles et un taux d'humidité du sol élevés avec présence de cours d'eau et de basfonds, les localités sont proche des points d'eau et les altitudes sont basses. Elle 
constitue une zone à risque ou vulnérables à la pathologie.

\section{Risque de l'ulcère de Buruli global}

La carte de risque de l'ulcère de Buruli est issue de la combinaison linéaire des cartes de risque climatique, environnementale et la carte d'occupation du sol selon leur poids respectif. L'observation de cette carte (Figure 4) montre que la partie sud, à proximité du lac de Kossou et la zone du Parc de la Marahoué sont plus vulnérable à la transmission de la maladie avec un degré de risque élevé couvrant $57,17 \%$ de la région. Cette partie se trouve en zone pré-forestière caractérisé par une pluviométrie abondante, des indices de végétation et d'humidité du sol très élevé avec une forte présence de lacs naturels, de zones marécageuses et de bas-fonds rizicoles à cause de sa proximité avec la région des lacs (Yamoussoukro). L'écoulement des eaux pluviales devient lent à cause de la présence des zones marécageuses et des bas-fonds

La classe de risque faible couvre $42,83 \%$ et se situe au nord de la région. La pluviométrie de cette zone est faible et les altitudes sont élevées ce qui favorise un écoulent rapide des eaux de pluie. La végétation est moins dense, avec une faible présence de lac et de bas-fond et les cultures dans cette région sont caractérisées par des cultures industrielles.

\section{Evaluation des incertitudes des cartes}

La validation des cartes thématiques s'est faite par l'analyse statistique de l'erreur ou de l'incertitude associée à chaque carte (Tableau 4). Les incertitudes calculées sur les facteurs d'évaluation du risque de l'ulcère de Buruli, varient plus ou moins de 0,004 à 0,005. Ainsi, les erreurs commises dans l'élaboration de ces cartes sont minime. Le niveau de confiance des différentes cartes de risque à l'ulcère de Buruli est significatif (95\%.).

Pour la carte d'occupation du sol, l'incertitude est de 3E-4 avec un intervalle de confiance de $68 \%$. Ce qui signifie que la carte d'occupation du sol n'est pas une bonne approximation des zones à risque de cette pathologie.

Tableau 1 : Classification et standardisation des critères de décision.

\begin{tabular}{|c|c|c|c|c|c|}
\hline Indicateur & Critères & Paramètres & Valeurs & Côtes & Classes \\
\hline \multirow{8}{*}{ Environnemental } & \multirow{2}{*}{ Couvert végétal } & \multirow{2}{*}{ ndvi } & $<-0,02$ et $>0,41$ & 1 & risque faible \\
\hline & & & de $-0,02$ à 0,41 & 2 & risque élevé \\
\hline & \multirow{2}{*}{ humidité du sol } & \multirow{2}{*}{ ndwi } & $<-0,2$ et $>0,94$ & 1 & risque faible \\
\hline & & & de $-0,2$ à 0,94 & 2 & risque élevé \\
\hline & \multirow{2}{*}{ Hydrographie } & \multirow{2}{*}{ distance en tout point } & $>1,5 \mathrm{~km}$ & 1 & risque faible \\
\hline & & & $<1,5 \mathrm{~km}$ & 2 & risque élevé \\
\hline & \multirow{2}{*}{ relief } & \multirow{2}{*}{ altitude } & $>137 \mathrm{~m}$ & 1 & risque faible \\
\hline & & & $<137 \mathrm{~m}$ & 2 & risque élevé \\
\hline \multirow{2}{*}{ Démographie } & \multirow{2}{*}{ Population } & \multirow{2}{*}{ densité de population } & $<98,08$ & 1 & risque faible \\
\hline & & & $>98,09$ & 2 & risque élevé \\
\hline \multirow{6}{*}{ Climatique } & \multirow{2}{*}{ précipitation } & \multirow{2}{*}{ Hauteur de pluie annuelle } & $<1174 \mathrm{et}>1275$ & 1 & risque faible \\
\hline & & & 1174 à 1275 & 2 & risque élevé \\
\hline & \multirow{2}{*}{ Température } & \multirow{2}{*}{ Valeur de la température } & $<20,8$ et $>32,30$ & 1 & risque faible \\
\hline & & & 20,8 à 32,30 & 2 & risque élevé \\
\hline & \multirow{2}{*}{ Humidité relative } & \multirow{2}{*}{ Taux d'humidité } & $>78,06$ & 1 & risque faible \\
\hline & & & $<78,06$ & 2 & risque élevé \\
\hline
\end{tabular}


Tableau 2: Expression verbale et numérique de l'importance relative de critères (El Morjani, 2003).

\begin{tabular}{ll}
\hline Expression d'un critère par rapport à un autre & Note \\
\hline Même importance & 1 \\
Modérément important & 3 \\
Fortement important & 5 \\
Très important & 7 \\
Extrêmement important & 9 \\
Extrêmement moins important & $1 / 3$ \\
Fortement moins important & $1 / 5$ \\
Moins important & $1 / 7$ \\
Extrêmement moins important & $1 / 9$ \\
\hline
\end{tabular}

Tableau 3 : Coefficient de pondération des facteurs de risque pour l'ulcère de Buruli (UB).

\begin{tabular}{lcc}
\hline Paramètres & $\begin{array}{c}\text { Vecteur propre } \\
(\mathbf{V P})\end{array}$ & Coefficient de pondération (CP \\
\hline pluie & 0.67 & 0.13 \\
T max & 2.94 & 0.55 \\
T min & 1.32 & 0.25 \\
HR & 0.39 & 0.07 \\
FC & $\mathbf{0 . 3 6}$ & $\mathbf{0 . 0 8}$ \\
NDWI & 1.72 & 0.27 \\
NDVI & 1.00 & 0.16 \\
Pop & 0.34 & 0.05 \\
altitude & 0.64 & 0.10 \\
hydro & 2.67 & 0.42 \\
FE & $\mathbf{3 . 2 7}$ & $\mathbf{0 . 7 3}$ \\
OCS & $\mathbf{0 . 8 4}$ & $\mathbf{0 . 1 9}$ \\
\hline \multicolumn{2}{c}{ FC : Facteurs Climatique $;$ FE : Facteurs environnementaux ; OCS : Occupation du sol }
\end{tabular}

Tableau 4 : Statistique des critères d'évaluation des facteurs de risque.

\begin{tabular}{|c|c|c|c|c|c|c|c|c|}
\hline Paramètres & $\max$ & $\min$ & moyenne & Ecart-Type & Totale & Incertitude & $\mathbf{K}$ & NC \\
\hline OCS & 5 & 1 & 4.07 & 1.32 & 21760440 & 0.0003 & 1 & 68 \\
\hline Risque d'UB & & & & & & & & \\
\hline Sensibilité au climat & 4.6 & 1.93 & 3.1 & 0.82 & 40504 & 0.004 & 2 & 95 \\
\hline Sensibilité écologique & 4.96 & 1 & 2.96 & 0.78 & 40818 & 0.004 & 2 & 95 \\
\hline $\begin{array}{l}\text { Carte de risque de } \\
\text { l'UB }\end{array}$ & 5 & 1 & 3.05 & 1.03 & 39569 & 0.005 & 2 & 95 \\
\hline
\end{tabular}



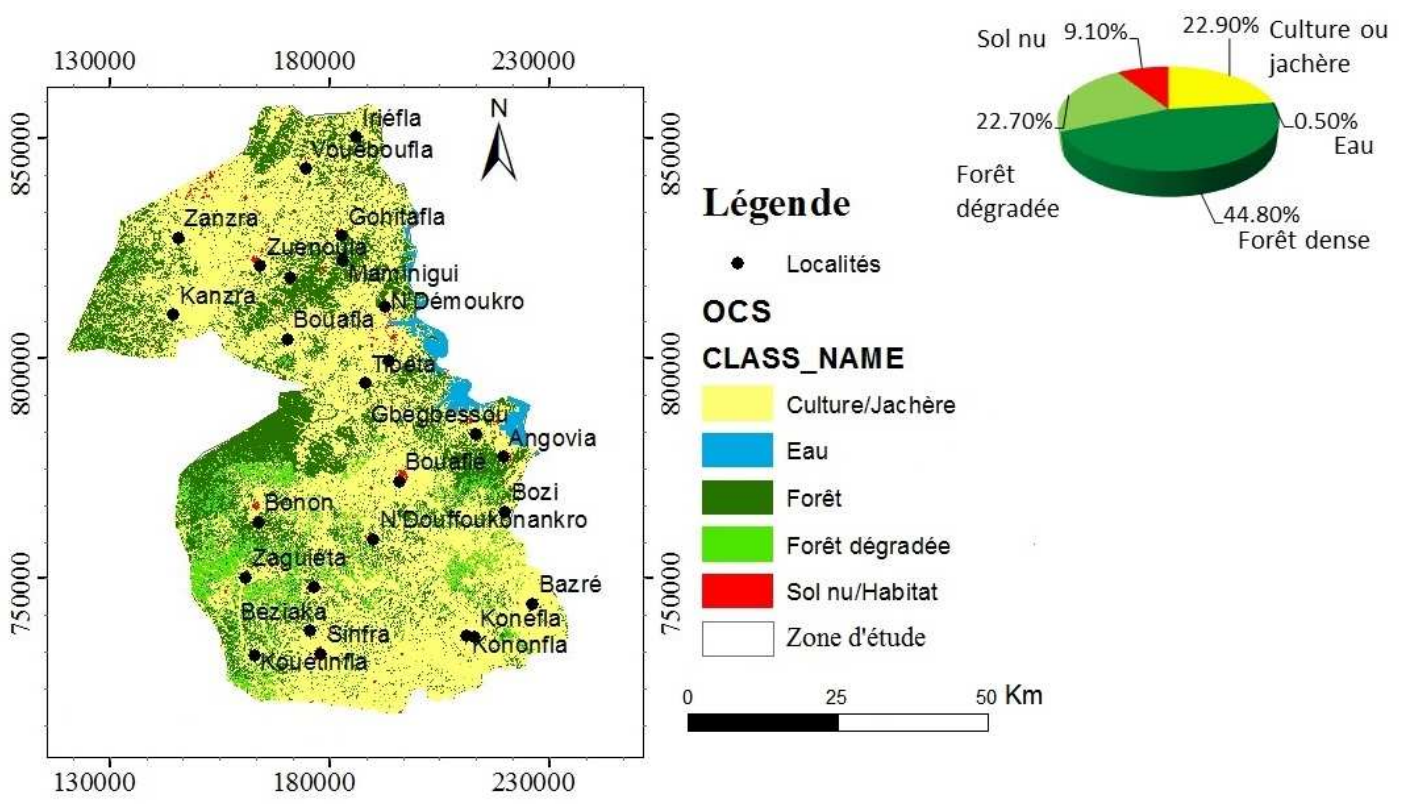

Figure 1 : Carte d'occupation du sol.

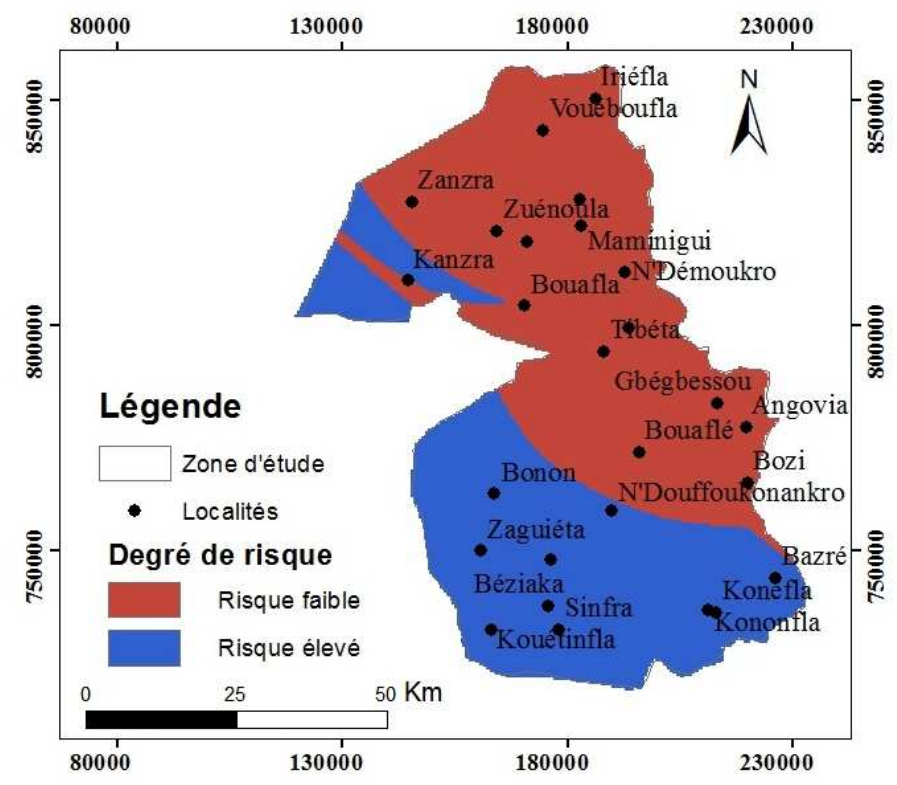

Figure 2: Carte de risque de l'ulcère de Buruli en fonction des facteurs climatiques. 


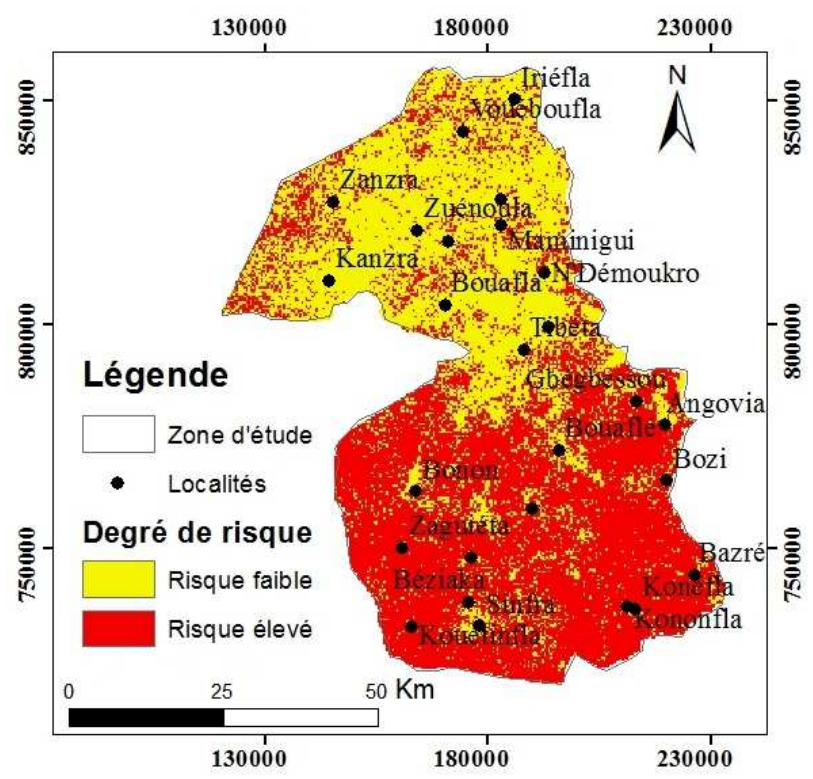

Figure 3: Carte risque de l'ulcère de Buruli en fonction des facteurs environnementaux.

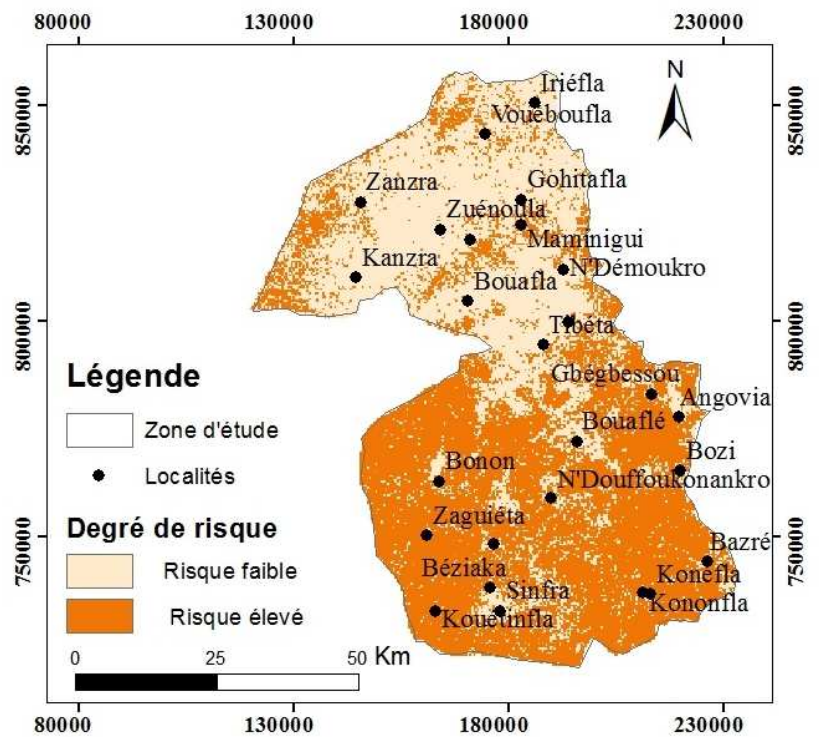

Figure 4: Carte de risque de l'ulcère de Buruli.

\section{DISCUSSION}

L'utilisation

des

systèmes d'information géographique (SIG) et l'analyse multicritère dans la région de la Marahoué ont abouti à la production des cartes du risque climatique, les cartes du risque environnementales et la carte d'occupation du sol. La combinaison linéaire de ces cartes a permis d'identifier les zones potentielles permettant de prédire le risque de l'ulcère de Buruli dans cette région. La carte de risque de l'ulcère de Buruli due aux facteurs climatiques possède un risque élevé de prédire cette maladie dans la région $(41,54 \%$ de la 
superficie totale). Ce risque élevé est plus marqué au sud de la région caractérisée par des précipitations abondantes, un taux d'humidité élevé et des températures max de plus de $30{ }^{\circ} \mathrm{C}$. Ce résultat montre que la transmission de l'UB dans région, serait due à l'abondance des précipitations. En effet, l'abondance des précipitations peut induire des inondations qui favorisent la recrudescence de cette maladie (Kanga et al., 2004). Selon OMS (2000), des flambées d'épidémie qui sont apparu au tour des lacs en Ouganda entre 1962 et 1964 étaient liées à l'abondance des précipitations. Le risque climatique découle également de l'élévation des températures et de la réduction des précipitations comme observées dans le nord de la région. Lorsque les précipitations baissent, cela réduit les écoulements des eaux de surface et entraîne la multiplication des zones d'eaux stagnantes et de mares temporaires qui constituent des foyers de prolifération de vecteur de la maladie (Kanga et al., 2004 ; Baquev, 2014).

Le risque dû aux facteurs environnementaux est dominé par un degré de risque élevé. Ce risque environnemental est marqué au sud de la région et couvre 55,24\% de la superficie. Ce risque élevé est dû à la forte densité chlorophyllienne et au taux élevé de l'humidité du sol traduisant la présence de forêt et de végétaux. Selon Brou et al. (2006), les zones de forêt et de contact forêt savane semble fournir les meilleures conditions écologiques de développement de l'ulcère de Buruli. Dans leurs études ils ont montré que, les forts taux de prévalence d'UB en Côte d'ivoire sont notés dans la partie mésophile de la zone forestière. Le risque élevé d'UB est aussi lié à l'altitude et à l'hydrographie. En effet, en zone de basse altitude et à proximité des points d'eau (lacs, marais, cours d'eau, zone marécageuse, irrigué ou drainé), la transmission de la maladie est très importante (Kanga et al., 2000 ; Tricia et al., 2007).
La région de la marahoué est une zone forestière baignée par des cours d'eaux et des lacs avec un relief plat et monotone favorisant la présence de zones marécageuses comme des bas-fonds. La présence de ces bas-fonds et mares temporaires entraine une humidité permanente du sol qui favoriserait la présence de grands foyers (Brou et al., 2006). En effet, l'ulcère de Buruli est une maladie environnementale dont l'épidémiologie est lié à l'écosystème aquatique (Darie, 2003 ; Christine et al., 2012) et aux zones hydroagricoles(Brou et al., 2006).Le risque élevé est aussi perçu au tour du lac de Kossou. Ce résultat s'accorde avec les travaux de Kanga et al. (2004) et Brou et al. (2006) qui stipulent que les conditions environnementales dues à l'homme comme les barrages et lacs artificiels jouent un rôle majeur dans l'éclosion de la maladie en créant des zones faiblement drainées propices au développement de la maladie. Ainsi le contact homme-point d'eau par les pratiques agricoles comme la riziculture, la pêche, les baignades et la chasse peuvent favoriser la transmission (Kanga et al., 2000 ; Tricia et al., 2007). La région de la marahoué, est constituée en grande partie des cultures et la jachère. En effet, la déforestation et l'accroissement des activités agricoles pourrait aussi contribuer de manière importante à l'augmentation marquée de l'incidence (OMS 2000). Le calcul des incertitudes et du niveau de confiance a permis de déterminer les erreurs importantes faites sur les cartes de risque. Les paramètres de forte incertitude ont une importance moindre que les paramètres de faible altitude (Mangoua et al., 2014). Les incertitudes déterminées sur les cartes de risque climatique et environnemental sont faibles donnant une grande fiabilité des cartes $(95 \%)$. Ces faibles incertitudes pourraient être dues au fait que ces paramètres proviennent de source déjà validée donc plus fiable. 


\section{Conclusion}

La modélisation par analyse multicritère a permis d'établir la carte de risque de l'ulcère de Buruli. Deux classes de risque ont été identifiées : la classe de risque faible et la classe de risque élevé. Ces deux classes utilisées dans cette approche rendent le modèle plus souple. La démarche adoptée dans ce travail utilise les données climatiques et les données environnementales. Cette approche a de nombreux avantages puisqu'elle a permis de montrer que les zones à risque d'ulcère de Buruli sont des zones marécageuses, avec présence de bas-fonds, mares et de lacs. Ces zones sont caractérisées par une pluviométrie abondante, des températures maximales élevées avec présence de végétaux et un écoulement lent des eaux de pluie. Elle a contribué ainsi à la détermination des zones vulnérables à l''ulcère de Buruli afin de prédire cette maladie et à la prise de décision rationnelle. Cependant, ces méthodes ont des limites par ce que l'estimation des paramètres manque souvent de précision en raison de l'insuffisance ou totale absence de donnée dans certaines parties de la zone d'étude. Les SIG demeurent une contribution inestimable dans la gestion de la santé humaine.

\section{CONFLIT D'INTERETS}

Les auteurs déclarent qu'ils n'ont aucun conflit d'intérêts.

\section{CONTRIBUTIONS DES AUTEURS}

Chaque auteur a contribué de manière significative à la réalisation du travail et de l'article. PGA a contribué à la rédaction de l'article ; ESG a contribué aux traitements des données ; AKZ a contribué à la réalisation des cartes ; AGBT a contribué à la lecture et la supervision de l'article.

\section{REMERCIEMENTS}

Les auteurs remercient le ministère de la santé et de l'hygiène publique en Côte d'ivoire pour les données sanitaires fourni et la Société d'Exploitation et de Développement Aéroportuaire, Aéronautique et Météorologie (SODEXAM) de Côte d'Ivoire pour la mise à disposition des données climatiques.

\section{REFERENCES}

Baquev. 2014. Ulcère de Buruli : le changement climatique provoque une augmentation des cas en Guyane: sciences :

http://www.la1ere.fr/2014/09/16/ visité $19 / 11 / 2015$

Beale L., Abellan J.J., Hodgson S. et Jarup L. 2008. Methodologic issues and approaches to spatial epidemiology. Environ Health Perspect, 116(8):11051110.

Brou T, Broutin H, Elguero E, Asse H, Guegan JF. 2008. Landscape diversity related to Buruli ulcer disease in Côte d'Ivoire. PLoSNegl Trop Dis, 2(7): e271. Doi : 10.1371/journal.pntd.0000271

Brou T, Broutin H, Dosso M, Guegan JF. 2006. Impact des modifications d'habitats (déforestation, aménagements hydroagricoles, extension des cultures) sur la santé des populations rurales: cas de l'ulcère de Buruli en Côte d'Ivoire. World Conference held at Havana, Cuba : IAHSPubl, 308.

Christinet V, Di BenedettoC, Comte E, Calmy A. 2012. Buruli et VIH : une interaction complexe et peu étudiée. Médecine Tropicale. Rev Med Suisse, 8: 1792-3p.

Darie H. 2003. Infection par Mycobacterium ulcerans : aspects épidémiologiques cliniques et thérapeutiques. Bull Soc Pathol Exot (SPE), 96(5) : 368-371.

El Morjani Z. 2003. Conception d'un système d'information à référence spatiale pour la gestion environnementale ; application à la sélection de sites potentiels de stockage de déchets ménagers et industriels en région semi-aride (Souss, Maroc). Thèse de Doctorat, Université de Genève. 
Fener P. 2009. Ulcère de Buruli et infection à VIH : http://sidasciences.inist.fr/?Ulcerede-Buruli-et-infection-téléchargé le $19 / 11 / 2015$

Kibadi K.2005. Ulcère à Mycobacterium ulcerans: Prise en charge chirurgicale dans 102 observations en République Démocratique du Congo. Med Trop, 65: 444-448.

Kanga JM,Kacou ED, Kouamé K, Kassi E, Kaloga M, Yao JK, Dion-Laine M, Avoaka LE, YobouéYP, Sangaré A, Ecra JE, Ahogo C, Djedje MS, Kadiri AJ, Aye C. 2004. L'ulcère de Buruli ; Aspect épidémiologique, cliniques et thérapeutiques en Côte d'ivoire. Med Trop, 64 : 238-242.

Kanga JM, Dion-Laine M, Kacou DE, Menan EIH. 2000. L'apport de l'héparinothérapie dans le traitement médical de l'ulcère de Buruli, à propos d'une observation. Bull Soc Pathol Exot, $94: 32-35$.

Kanga JM, Kacou ED. 2000. Aspects épidémiologiques de l'ulcère de Buruli en Côte d'Ivoire: résultats d'une enquête nationale. Santé Publique, 2193 : 6p.

Kouamé AKD, Mobio ABH, Djagoua EMV, Affian K, Pottier P. 2011. Cartographie $\mathrm{du}$ risque bilharzienne à partir de l'utilisation combinée de la télédétection et du système d'information géographique.

http://icaci.org/files/documents/ICC_proc eedings/ICC2011http://icaci.org/files/doc uments/ICC_proceedings/ICC2011, 18p.

Kouamé 2010. Facteurs de répartitions et essaie de modélisation des Schistosomes et des géoherminthes par des techniques de télédétections et du système d'information géographique pour un développement durable dans la région de l'Agnéby. Thèse unique de doctorat en Télédétection et les systèmes d'information géographique. Université de Cocody, $164 \mathrm{p}$.
Mangoua M J1, Dibi B, Koblan EW, Douagui GA, Kouassi KA, Savané I, Biémi J. 2014. Map of potential areas of groundwater by the multi-criteria analysis for the needs for water of the Baya's catchment basin (East of Côte d'Ivoire). African Journal of Agricultural Research, 9(45): 3319-3329.

Marsollier L, Aubry J, Saint-Andre JP, Robert R, Legras P, Manceau AL, Bourdon S, Audrain C, Carbonnelle B. 2003. Ecology and transmission of Mycobacterium ulcerans. Pathol. Biol. (Paris), 51 : 490495.

Martin JL, Henry E, Boulemia C, Masson FX. 2004. Proposition d'outils de gestion et d'aide à la décision dans une moyenne collectivité locale : application à l'exploitation de la ressource en eau souterraine. Acte de la journée d'études "Les territoires de l'eau », Université d'Artois. Arras, 26 mars 2004, 74-82. 7482.

Meyer-Waarden L,Zeitoun H. 2005. Une comparaison empirique de la validité prédictive de la méthode de composition, de l'analyse conjointe et de l'analyse conjointe hybride. Recherche et Applications en Marketing, 20(3) : 39-58.

Mouchou, Mougin B. 2009. L'ulcère de Buruli en pays tropicale : Etat des lieux. DES de Médecine Générale à l'Université Paul Sabatier de Toulouse ; 1-50.

OMS. 2015. Ulcère de Buruli Infection à Mycobacterium ulcerans. Centre des Médias. $\quad$ Aide-mémoire $\quad \mathrm{N}^{\circ} 199$. http://www.who.int/mediacentre/factsheet s/fs199/fr/

OMS. 2004. Rapporte de la septième réunion du groupe consultatif spécial de l'OMS sur l'Ulcère de Buruli. http://apps.who.int/iris/bitstream/10665/6 8996/1/WHOCDS GBUI 20049 fre.pdf

OMS. 2000. Ulcère de Buruli: Infection à Mycobacterium ulcerans. Who/cds/cpe/gbuil ; 118 p. 
Saaty TL. 1977. A scaling method for priorities in hierarchical structures. Journal of Mathematical Psychology, 15 : 234-281.

Somé YCS. 2010. Modélisation de la distribution spatiale des formes moléculaires $M$ et $S$ d'Anophèles gambiae Sensu Stricto au Burkina Faso avec les SIG et l'analyse spatiale. Thèse de Doctorat à l'Université d'Orléans : Spécialité ; Géographie-AménagementEnvironnement.

Tricia YJQ, Athan E, Margaret JH, Pasco JA, Redden-Hoare J, Hughes A, Johnson PDR. 2007. Risk Factors for Mycobacterium ulcerans Infection, Southeastern Australia. Emerging Infectious Diseases, 13(11): 1661-1666. www.cdc.gov/eid
Yao AB, Goula BTA, Kane A, Mangoua OMJ, Kouassi KA. 2014. Cartographie du potentiel en eau souterraine du bassin versant de la Lobo (Centre-Ouest, Côte d'Ivoire). Approche par analyse multicritère. Hydrological Sciences Journal : 23p. DOI: 10.1080/02626667.2014.932360

Youan Ta M, Lasm T, Jourda JP, Saley BM, Adja MG, Kouamé K, Biemi J. 2011. Cartographie des eaux souterraines en milieu fissuré par analyse multicritère. Cas de Bondoukou (Côte-d'Ivoire). Revue Internationale de Géomatique, 21(1) : 43-71. doi:10.3166/RIG.21.4371. 